\title{
Light deflection in binary stars
}

\author{
Sven Zschocke \\ Lohrmann Observatory, \\ Dresden Technical University, \\ Mommsen Str. 13, \\ D-01062 Dresden, Germany
}

The light deflection of one component of a binary system due to the gravitational field of the other component is investigated. While this relativistic effect has not been observed thus far, the question arises that whether this effect becomes detectable in view of todays high-precision astrometry which soon will reach the microarcsecond level of accuracy. The effect is studied and its observability is investigated. It turns out, that in total there are about $10^{3}$ binaries having orbital parameters such that the light deflection amounts to be at least 1 microarcsecond. Two stringent criteria for the orbital parameters are presented, by means of which one can easily determine the maximal value of light deflection effect for a given binary system. It is found, that for relevant binaries their orbital parameters must take rather extreme values in order to have a light deflection of the order of a few microarcseconds. Only in a very few and rather extreme binary systems the light deflection effect might be detectable by todays astrometry, but their existence is highly improbable. Thus, the detection of this subtle effect of relativity still remains a challenge for future astrometric missions. 


\section{Contents}

I. Introduction 3

II. Orbital elements of a binary system

III. Inclination formula from generalized lens equation 7

IV. Stringent conditions on orbital parameters for binary systems 10

V. Total number of binaries with a given light deflection for infinite time of observation

VI. Total number of binaries with a given light deflection for finite time of observation

VII. Special case: conditions on orbital parameters for resolved binaries

A. Resolving power of Gaia

15

B. Orbital Parameters of Resolved Binaries Observable by Gaia

VIII. Summary

Acknowledgements

References

A. Two-body problem

B. Derivation of Eq. (10)

C. Derivation of Eq. (13)

D. Derivation of Eq. (3)

E. Probability Distribution 


\section{INTRODUCTION}

Astrometric space missions, especially the ESA (European Space Agency) cornerstone mission Gaia, see e.g. Perryman, et al. [2001], are in preparation to attain microarcsecond ( $\mu$ as) level of accuracy in absolute positional measurements of stars and other celestial objects. This unprecedented accuracy of astrometric observations makes it necessary to account for many subtle effects which were totally negligible before. A practical model for astrometric observations with an accuracy of $1 \mu$ as has been formulated by Klioner [2003], where the influence of the gravitational fields inside the solar system were taken into account. Furthermore, a number of additional effects potentially observable at this level of accuracy due to various gravitational fields generated outside of the solar system were also briefly discussed in that investigation. One of them is the gravitational light deflection of one companion of a binary system in the gravitational field of the other companion (without loss of generality, throughout the investigation the light deflection of component $\mathrm{B}$ at component $\mathrm{A}$ is considered, hence, component $\mathrm{A}$ is considered to be the massive body, while component $\mathrm{B}$ is the light-source). This effect would change the apparent position of one component of a binary system. While it is clear that this effect is relatively small and even at the level of $1 \mu$ as observable only for edge-on binary systems, the huge amount of binaries generate some hope that there are relevant systems where this light deflection effect becomes detectable. For instance, Gaia will observe $10^{9}$ stars brighter than $20^{\text {th }}$ apparent magnitude. Detailed numerical simulations predict the detection of about $10^{8}$ (resolved, astrometric, eclipsing, spectroscopic) binary systems by Gaia mission, see Zwitter \& Munari [2004], which is a considerable increase compared to the $10^{5}$ binary systems known so far, see "Washington Double Star Catalog" 2011].

Let us consider this argument a bit more quantitatively. For a simple estimate of the expected order of magnitude in light deflection, the classical lens equation (in the form given by Eq.(67) in Fritelli, et al. [2000], Eq. (24) in Bozza [2008] or Eq. (23) in Zschocke [2011]) is applied. In terms of orbital elements of a binary system it can be written as follows:

$$
\varphi=\frac{1}{2}\left(\sqrt{\frac{A^{2}}{r^{2}} \cos ^{2} i+16 \frac{m}{r} \frac{A}{r} \sin i}-\frac{A}{r}|\cos i|\right) .
$$

Here, $\varphi$ is the light deflectin angle, $i$ is the inclination, $A$ is the semi-major axis and $r$ is the distance of center-of-mass of the binary system from the observer, and the Schwarzschild radius is $m=\frac{G M}{c^{2}}$, where $G$ is the gravitational constant and $c$ is the speed of light, and $M$ is the stellar mass of component A of the binary system. From (11) one obtains the maximal possible light deflection for edge-on binaries, i.e. attained for the case when the inclination is exactly $90^{\circ}$ :

$$
\varphi \leq 200 \mu \mathrm{as} \sqrt{\frac{M}{M_{\odot}} \frac{A}{\mathrm{AU}}} \frac{\mathrm{pc}}{r}
$$

Here, $\mathrm{AU}=1.496 \times 10^{11} \mathrm{~m}$ is the astronomical unit, $\mathrm{pc}=3.086 \times 10^{16} \mathrm{~m}$ stands for parallax of one arcsecond, and $M_{\odot}$ is the solar mass. According to this formula, the choice of moderate values like $M=M_{\odot}$ and $A \sim 100 \mathrm{AU}$ would result into significant light deflection effect on microarcsecond level even at large distances of about $r \sim 100 \mathrm{pc}$. A meaningful value for the density of stars in the solar neighborhood, 0.025 binaries $\mathrm{pc}^{-3}$, implies already $10^{5}$ 
binaries inside a sphere of $r=100 \mathrm{pc}$. Thus, one might conclude among them there are a few relevant edge-on binary systems.

However, in order to estimate quantitatively the number of relevant systems, one needs to know the probability for the occurrence of such edge-on binaries which have a given light deflection depending on their orbital parameters like inclination, mass, distance and semimajor axis. Such a relation between a given light deflection and orbital parameters is given by a so-called inclination formula. Since the inclinations of binary systems are of course randomly distributed, it is meaningful to resolve such an inclination formula in terms of inclination. As it has been shown by Klioner, et al. [2003] (see Appendix D for some basic steps), such an inclination formula can be obtained by means of the analytical solution of light deflection in standard post-Newtonian approach, and is given as follows:

$$
\left|\frac{\pi}{2}-i\right|_{\mathrm{KMS}} \leq 2 \arctan \left(0.0197 \frac{M}{M_{\odot}} \frac{\mu \mathrm{as}}{\varphi} \frac{\mathrm{pc}}{r}\right) \text {. }
$$

For a better illustration of the inclination formula, relation (3) is rewritten in terms of angular degrees instead of radians:

$$
\left|90^{\circ}-i\right| \leq 2.25^{\circ} \frac{M}{M_{\odot}} \frac{\mu \mathrm{as}}{\varphi} \frac{\mathrm{pc}}{r},
$$

where also $\arctan x=x+\mathcal{O}\left(x^{3}\right)$ has been used. According to this relation, the inclination $i$ of a binary system with stellar mass $M$ and at distance $r$ must not deviate from the edge-on value $90^{\circ}$ too much in order to have a given light deflection $\varphi$. For example, for a hypothetical binary star with $M=M_{\odot}$ situated at a distance of $r=10 \mathrm{pc}$ the light deflection effect attains $1 \mu$ as only if $\left|90^{\circ}-i\right|<0.225^{\circ}$, which means that the probability to observe this binary at a favorable inclination is only about $0.2 \%$.

But even this estimation is still much too optimistic. As a concrete example of todays high-precision astrometry, let us consider one important parameter about the astrometric accuracy of the Gaia mission: the accuracy of one individual positional measurement in the most ideal case (bright star, i.e. $10^{\text {th }}$ magnitude) amounts to be $25 \mu$ as, which implies $\varphi \geq$ $25 \mu$ as. Furthermore, inside a sphere of $10 \mathrm{pc}$ around the Sun almost every star and binary system is known already by the data of Research Consortium on Nearby Stars (RECONS) [2012]. Since inside that sphere there is no binary system having a light deflection on microarcsecond level, one has to take at least $r>10 \mathrm{pc}$. By taking into account these both remarks, one obtains $\frac{\mu \mathrm{as}}{\varphi} \frac{\mathrm{pc}}{r}=\frac{1}{250}$ in relation (4). Therefore, even in the best case one has to conclude $\left|90^{\circ}-i\right| \leq 0.01^{\circ} M / M_{\odot}$, that means (for $M=M_{\odot}$ ), the probability to observe such a binary at a favorable inclination is practically only about $0.01 \%$. And the binaries must be, in fact, almost edge-on in order to have a light deflection which can be detected by todays astrometry. Accordingly, while relation (2) triggers the hope about the existence of many relevant binary systems, from relation (4) one concludes that the number of relevant binary systems is considerably reduced.

An estimation of the total amount of binaries depends on many different parameters, like mass, semi-major axis, inclination and distances of the binaries. Therefore, a simple estimation is not so straightforward as one might believe. Moreover, the relation (2) has been obtained with the aid of classical lens equation, while relation (4) has been obtain by means of standard post-Newtonian approach. These both approaches have different regions of validity. However, a rigorous treatment of the problem of light deflection in binary systems 
implies the need of an analytical formula which is valid for such kind of extreme astrometric configurations like the binary systems are. Recently, a generalized lens equation has been derived by Zschocke [2011], which allows to determine the light deflection of binary systems on microarcsecond level. One aim of this study is, therefore, to reobtain the criteria (2) and (44) as stringent conditions from one and the same approach, i.e. with the aid of generalized lens equation. This is possible, because in the corresponding limits the generalized lens equation agrees with the classical lens equation and the standard post-Newtonian solution. Another aim is, to derive an inclination formula like (3) for binary systems which allows to determine the needed inclination for a given light deflection angle and as a function of the orbital parameters. For that one has to take into account the distribution of stellar masses and the distribution of semi-major axes in binary systems. Finally, the aim of this study is, to determine the total number of relevant binaries having a light deflection on microarcsecond level, and to investigate the possibility to detect this effect of light deflection by todays high precision astrometry.

The article is organized as follows: In Section [I] some basics about orbital elements of binary systems are given. The generalized lens equation and the inclination formula are presented in Section [II]. In Section [IV two stringent conditions on the orbital elements of binary systems (astrometric, spectroscopic, eclipsing and resolved binaries) are presented, which allow to determine whether or not the binary system will have a light deflection of a given magnitude. The total number of binaries which have a given light deflection for an infinite time of observation is estimated in Section $\nabla$, while the more practical case of a finite time of observation is considered in Section VI. The special case of resolved binaries is considered in Section VII. For that, the specific instrumentation of Gaia mission is considered in some detail as the most modern astrometric mission with todays highest possible accuracy. A Summary is given in Section VIII.

\section{ORBITAL ELEMENTS OF A BINARY SYSTEM}

Consider a binary system, component $A$ with mass $M_{A}$ at coordinate $\boldsymbol{r}_{A}$ and component $B$ with mass $M_{B}$ at coordinate $\boldsymbol{r}_{B}$. In order to express the light deflection effect in terms of orbital elements, spherical coordinates are introduced, illustrated by FIG. 1 .

The center of coordinate system is located at the CMS, i.e.

$$
\boldsymbol{r}_{\mathrm{CMS}}=\frac{1}{M_{A}+M_{B}}\left(M_{A} \boldsymbol{r}_{A}+M_{B} \boldsymbol{r}_{B}\right)
$$

Thus, the vector $\boldsymbol{r}$, which points from CMS to the observer, is given by

$$
\boldsymbol{r}=\left(\begin{array}{c}
r \cos \omega \sin i \\
r \sin \omega \sin i \\
r \cos i
\end{array}\right)
$$

where $r=|\boldsymbol{r}|$, the argument of periapsis is denoted by $\omega$, and $i$ is the inclination, see FIG. 1. The solution of equation of motion yields for vectors $\boldsymbol{r}_{A}$ and $\boldsymbol{r}_{B}$ the expression given by Eqs. (A22) - (A26). The vector $\boldsymbol{x}_{1}$ points from the mass center of massive body to the observer, and vector $\boldsymbol{x}_{0}$ points from the mass center of massive body to the source, see 


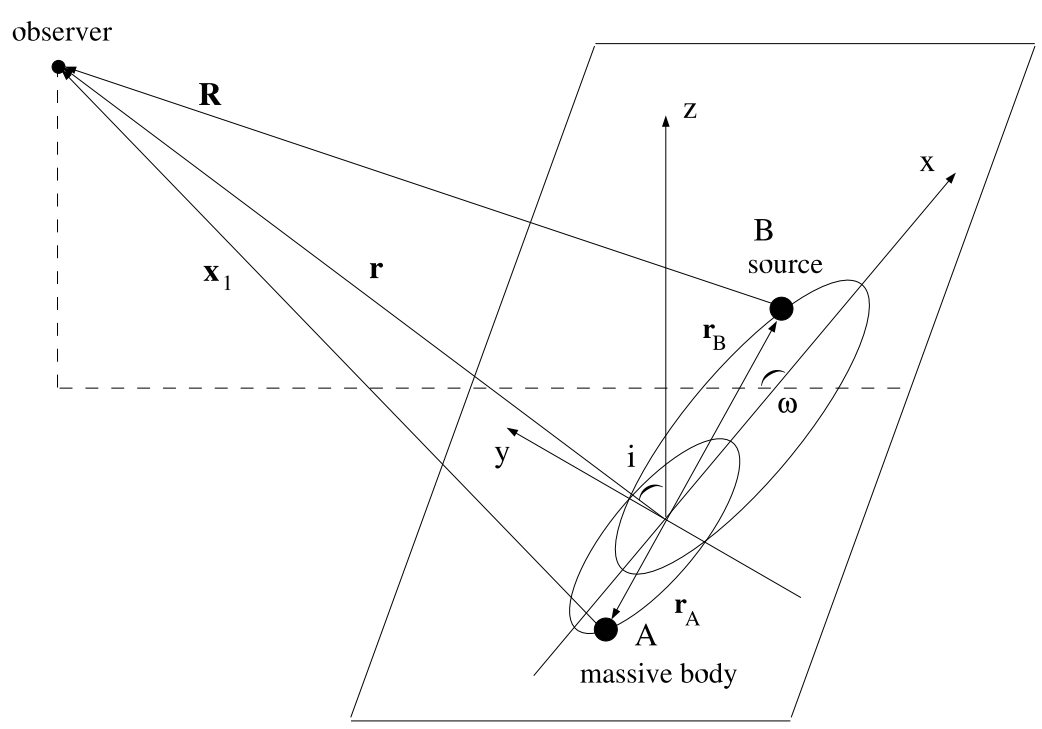

FIG. 1: Seven orbital elements which define the orbit of a binary system: distance vector $\boldsymbol{r}$, semi-major axis $A$, inclination $i$, eccentricity $e$, eccentric anomaly $E$, periapsis $\omega$, and mass ration $M_{A} / M_{B}$. The orbit of the binary system spans the $(x, y)$-plane and the $z$-axis is perpendicular to the orbital plane. The $x$-axis is oriented along the semi-major axis of the orbit of the binary system, while the $y$-axis is perpendicular to the $x$-axis. The vector $\boldsymbol{r}$ is directed from the center-ofmass (CMS) of the binary system, see Eq. (15), to the observer. The center of spherical coordinate system is located at the CMS of binary system, i.e. $\boldsymbol{r}_{\mathrm{CMS}}=\mathbf{0}$. The inclination $0 \leq i \leq \pi$ is the angle between $\boldsymbol{r}$ and $z$-axis; $i=\pi / 2$ is called edge-on and $i>\pi / 2$ corresponds to retrograde orbit. The dotted line indicates the projection of $\boldsymbol{r}$ onto orbital $(x, y)$-plane, i.e. z-component of $\boldsymbol{r}$ equals zero. The angle between this projection and $x$-axis is called argument of periapsis $0 \leq \omega \leq \pi$. The orbital elements semi-major axis $A$, eccentricity $0 \leq \mathrm{e} \leq 1$ and mass ratio $M_{A} / M_{B}$ govern uniquely the geometric shape of both ellipses. The eccentric anomaly $0 \leq E \leq 2 \pi$ (not plotted here), is defined in Eq. (A16) of Appendix $\mathrm{A}$ and determines the actual position of the bodies $\mathrm{A}$ and $\mathrm{B}$ on their orbit.

also FIG. 1, The coordinates of these vectors can be expressed by the orbital elements of the binary star as follows:

$$
\boldsymbol{x}_{1}=\boldsymbol{r}-\boldsymbol{r}_{\mathrm{A}}=\left(\begin{array}{c}
r \cos \omega \sin i-\frac{A(\cos E-\mathrm{e})}{1+\frac{M_{A}}{M_{B}}} \\
r \sin \omega \sin i-\frac{A \sqrt{1-\mathrm{e}^{2}} \sin E}{1+\frac{M_{A}}{M_{B}}} \\
r \cos i
\end{array}\right),
$$




$$
\boldsymbol{x}_{0}=\boldsymbol{r}_{\mathrm{B}}-\boldsymbol{r}_{\mathrm{A}}=-\left(\begin{array}{c}
A(\cos E-\mathrm{e}) \\
A \sqrt{1-\mathrm{e}^{2}} \sin E \\
0
\end{array}\right) .
$$

Here, $A$ is the semi-major axis, $e$ is the eccentricity, and $E$ is the eccentric anomaly, see Appendix A. The vectors (7) and (8) will be used to express the light deflection in terms of orbital elements of the binary system.

\section{INCLINATION FORMULA FROM GENERALIZED LENS EQUATION}

A scheme of light propagation of a signal emitted at component B in the gravitational field of component A is shown in FIG. 2. The vector $\boldsymbol{x}_{1}$ points from the mass center of massive body to the observer, and vector $\boldsymbol{x}_{0}$ points from the mass center of massive body to the source, and we define $\boldsymbol{R}=\boldsymbol{x}_{1}-\boldsymbol{x}_{0}$, the absolute value $R=|\boldsymbol{R}|$ and unit vector by $\boldsymbol{k}=\boldsymbol{R} / R$. Furthermore, the impact vector $\boldsymbol{d}=\boldsymbol{k} \times\left(\boldsymbol{x}_{1} \times \boldsymbol{k}\right)$ is defined, and its absolute value is denoted by $d=|\boldsymbol{d}|$. The Schwarzschild radius of massive body, i.e. of component A of the binary system is denoted by $m=\frac{G M}{c^{2}}$.

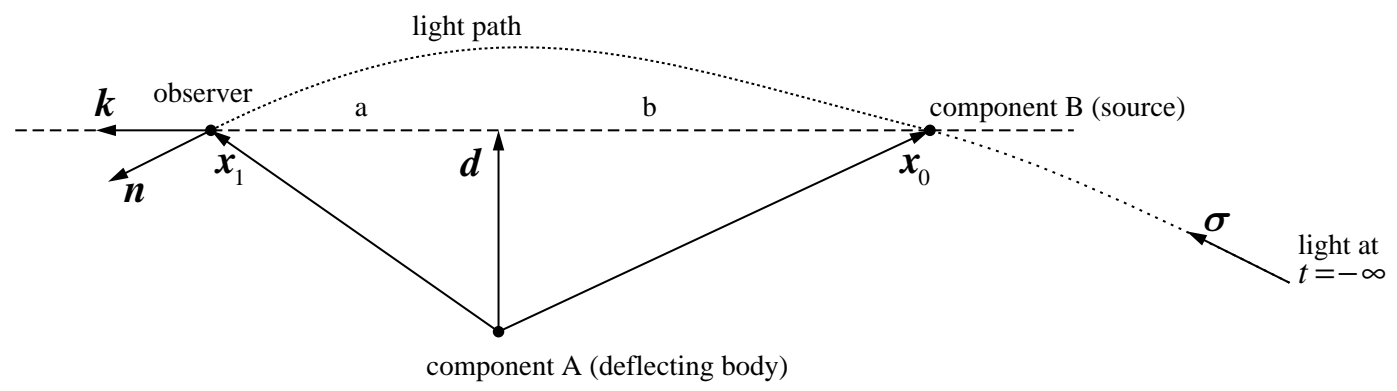

FIG. 2: Binary star composed of component A being the massive body, and component B considered to be the light-source.

For determining the light deflection in weak gravitational fields there are two essential approaches: standard post-Newtonian approach, e.g. Brumberg [1991], and classical lens equation e.g. Eq.(67) in Fritelli, et al. [2000], Eq. (24) in Bozza [2008], or Eq. (23) in $Z$ schocke [2011]. While the first approach is restricted by the condition $m \gg d$, the second approach is only valid for the case that source and observer are far from the massive body, especially for $a=\boldsymbol{k} \cdot \boldsymbol{x}_{1} \gg d$ and $b=-\boldsymbol{k} \cdot \boldsymbol{x}_{0} \gg d$ (for geometrical illustration of $a, b$ and $d$ see FIG. 2). However, in binary systems extreme configurations are possible like $d=0$ or $b=0$. Therefore, in order to investigate the light deflection in binary systems one needs a generalized lens equation which is valid in such extreme configurations where the standard post-Newtonian approach as well as the classical lens equation cannot be applied. Recently, Zschocke [2011] has derived a generalized lens equation, which allows to determine the light deflection in such extreme astrometric configurations like the binary systems are:

$$
\varphi=\frac{1}{2}\left(\sqrt{\frac{d^{2}}{x_{1}^{2}}+8 \frac{m}{x_{1}} \frac{x_{0} x_{1}-\boldsymbol{x}_{0} \cdot \boldsymbol{x}_{1}}{R x_{1}}}-\frac{d}{x_{1}}\right) .
$$


Actually, the lens equation has two solutions, but here only one solution is considered, while the second solution represents just the second image of one and the same source which is not relevant in our investigation. The generalized lens equation is valid up to terms of the order $\mathcal{O}\left(\frac{m^{2}}{d^{\prime 2}}\right)$, and the absolute value of their total sum can be shown to be smaller or equal to $\frac{15 \pi}{4} \frac{m^{2}}{d^{\prime 2}}$. Here, $d^{\prime}=\frac{L}{E}$ is Chandrasekhar's impact parameter, see Chandrasekhar [1983], where $L$ being the orbital momentum and $E$ is the energy of the photon in the gravitational field of massive body. Basically, the light-ray of component B cannot be observed if $d^{\prime}$ is smaller than the radius of massive body A. For stars, the radius is much larger than Schwarzschild radius $m$, hence $\frac{m^{2}}{d^{\prime 2}} \ll 1$. Furthermore, the generalized lens equation (9) is finite for $d \rightarrow 0$ and $b=-\boldsymbol{k} \cdot \boldsymbol{x}_{0} \rightarrow 0$, both of which are possible astrometric configurations in binary systems. Furthermore, in Zschocke [2011] it has been shown that the generalized lens equation (91) yields in the appropriate limits the correct standard post-Newtonian solution and the classical lens equation, hence provides a bridge between these essential approaches.

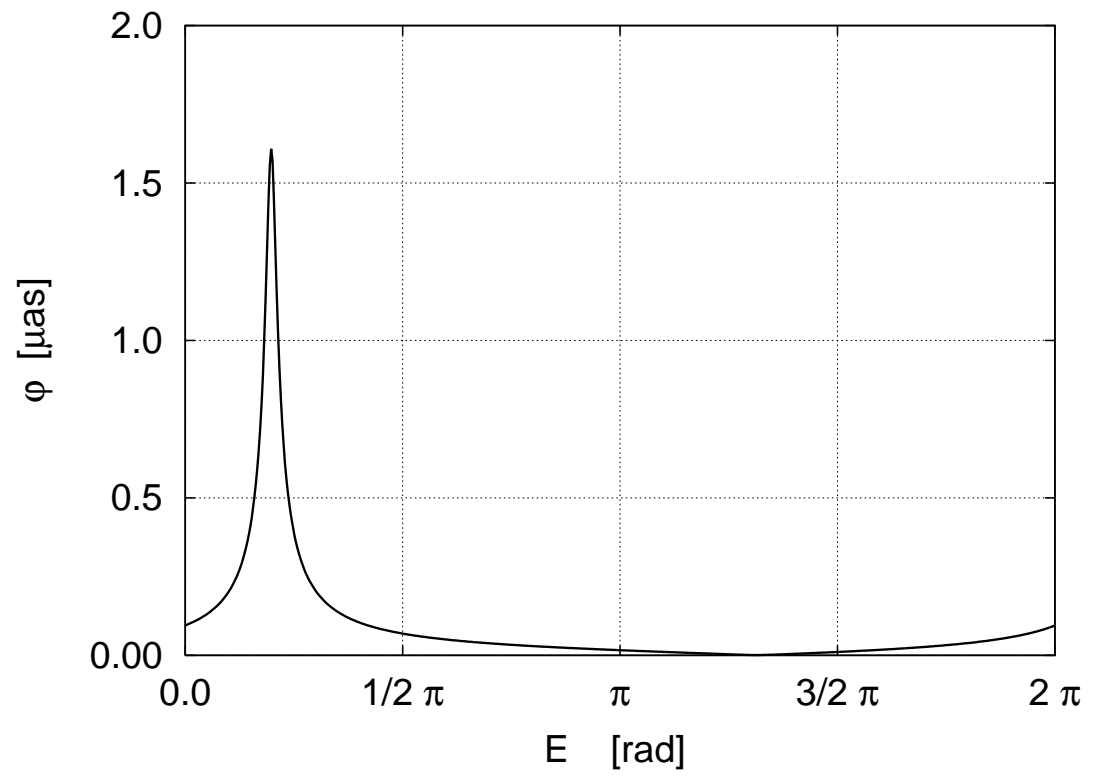

FIG. 3: Typical light curve of a binary system, determined using generalized lens equation (9) or (B11), respectively. The parameters chosen are: distance $r=1 \mathrm{pc}$, semi-major axis $A=100 \mathrm{AU}$ inclination $i=\frac{31}{64} \pi$, mass $M_{A}=2 M_{\odot}$, mass ratio $\frac{M_{A}}{M_{B}}=2.0$, eccentricity e $=0.25$, argument of periapsis $\omega=\frac{\pi}{4}$.

In the following, Eq. (9) is applied in order to determine the light deflection in binary systems. For that, the coordinates $\boldsymbol{x}_{0}$ and $\boldsymbol{x}_{1}$ are used in the form as given by Eqs. (7) and (8)), respectively. A typical light-curve of a binary system, calculated by means of generalized lens equation (9) is shown in FIG. 3 .

Now, an inclination formula is derived from the generalized lens equation (91). The impact of eccentricity is neglected, circular orbits e $=0$ are considered, implying $\omega=0$. Thus, the coordinates $\boldsymbol{x}_{0}$ and $\boldsymbol{x}_{1}$ are simplified to the expressions (B1) and (B2) given in Appendix B. Furthermore, the maximal value of light deflection of a binary system is of interest, i.e. the 
astrometric configuration $E=0$ is considered here. Then, by inserting these coordinates in the generalized lens equation (9) one obtains (see Eq. (B14) in Appendix B) up to terms of the order $\mathcal{O}\left(\frac{A}{r} \sqrt{\frac{m}{r} \frac{A}{r}}\right)$ :

$$
\varphi=\frac{1}{2}\left(\sqrt{\frac{A^{2}}{r^{2}} \cos ^{2} i+8 \frac{m}{r} \frac{A}{r}(1+\sin i)}-\frac{A}{r}|\cos i|\right) .
$$

The minimal value $\varphi_{\min }=\varphi(i=0)$ and maximal value $\varphi_{\max }=\varphi\left(i=\frac{\pi}{2}\right)$ of light deflection for the astrometric position $E=0$ follow from Eq. (10):

$$
\begin{aligned}
& \varphi_{\text {min }}=\frac{1}{2}\left(\sqrt{\frac{A^{2}}{r^{2}}+8 \frac{m}{r} \frac{A}{r}}-\frac{A}{r}\right) \approx 2 \frac{m}{r}=0.0197 \mu \mathrm{as} \frac{M}{M_{\odot}} \frac{\mathrm{pc}}{r}, \\
& \varphi_{\max }=2 \frac{\sqrt{m A}}{r}=200 \mu \mathrm{as} \sqrt{\frac{M}{M_{\odot}} \frac{A}{\mathrm{AU}}} \frac{\mathrm{pc}}{r},
\end{aligned}
$$

where in (11) terms of the order $\mathcal{O}\left(\frac{m^{2}}{r A}\right)$ have been neglected. The expression (10) can be reconverted in terms of inclination (see Appendix C):

$$
\left|\frac{\pi}{2}-i\right|=\arccos \left(-\frac{p}{2}+\sqrt{\frac{p^{2}}{4}-q}\right),
$$

where

$$
\begin{aligned}
& p=\frac{8 m^{2} A-4 m r^{2} \varphi^{2}}{A\left(r^{2} \varphi^{2}+4 m^{2}\right)} \\
& q=-\frac{A^{2} r^{2} \varphi^{2}+4 m A r^{2} \varphi^{2}-4 m^{2} A^{2}-r^{4} \varphi^{4}}{A^{2}\left(r^{2} \varphi^{2}+4 m^{2}\right)}
\end{aligned}
$$

The inclination formula (13) yields the upper limit for $\left|\frac{\pi}{2}-i\right|$ of a binary system in order to have a given value of light deflection $\varphi$. Note, that the values of $\varphi$ cannot be chosen arbitrarily, but they are restricted by $\varphi_{\min }$ and $\varphi_{\max }$ given by Eqs. (11) and (12), respectively.

The inclination formula (13) can considerably be simplified. From (14) and (15) one obtains by series expansion

$$
\begin{aligned}
& p=-4 \frac{m}{A}+8 \frac{m^{2}}{r^{2} \varphi^{2}}+\mathcal{O}\left(m^{3}\right) \\
& q=-1-4 \frac{m}{A}+8 \frac{m^{2}}{r^{2} \varphi^{2}}-4 \frac{m^{2}}{A^{2}}+\frac{r^{2} \varphi^{2}}{A^{2}}+\mathcal{O}\left(m^{3}\right) .
\end{aligned}
$$

By means of (12), the last term in (17) can be estimated to be smaller than $4 \frac{m}{A} \ll 1$. Here, it should be underlined that $\frac{m}{A} \ll \frac{m}{r \varphi}$ even at large distances $r \simeq 10^{3}$ pc and small values for semi-major axis $A \simeq 1$ AU. Thus, one obtains

$$
\left|\frac{\pi}{2}-i\right| \approx \arccos \left(1-8 \frac{m^{2}}{r^{2} \varphi^{2}}\right) \approx 2 \arctan \left(2 \frac{m}{r \varphi}\right)
$$


up to terms of the order $\mathcal{O}\left(\frac{m^{3}}{r^{3} \varphi^{3}}\right)$ and $\mathcal{O}\left(\frac{m}{A}\right)$; here the relation $\arccos \left(1-8 x^{2}\right)=$ $2 \arctan 2 x+\mathcal{O}\left(x^{3}\right)$ for $x \ll 1$ has been used. It should be underlined, that the applicability of (18) is restricted by the condition (D5) given in Appendix D and by $d \gg m$. Here, it should be noticed that $x=2 \frac{m}{r \varphi} \ll 1$, even in such an extreme case like $r=1 \mathrm{pc}, m=m_{\odot}$ and $\varphi \simeq 1 \mu$ as one obtains a small number $x=0.019$; for an analytical proof use the exact expression for $\varphi_{\min }$. Due to $\frac{m}{A} \ll \frac{m}{r \varphi}$, the impact of semi-major axis is of lower order and can be neglected in the inclination formula. The inclination formula (18) can be expressed in terms of dimensionless quantities as follows:

$$
\left|\frac{\pi}{2}-i\right| \approx 2 \arctan \left(0.0197 \frac{M}{M_{\odot}} \frac{\mu \mathrm{as}}{\varphi} \frac{\mathrm{pc}}{r}\right) .
$$

Note, that expression (19) agrees with an inclination formula (3) derived at the first time by Klioner, et al. [2003]; the arguments of their work are represented in Appendix D. The simplified inclination formula (19) is not only useful for straightforward estimations about the order of magnitude, but it's simple structure provides also an obvious comprehension about the interplay of the individual terms.

\section{STRINGENT CONDITIONS ON ORBITAL PARAMETERS FOR BINARY SYSTEMS}

In this Section, two stringent conditions on the orbital elements are highlighted for binaries having a given light deflection. These strict conditions are valid for any binary system: astrometric, spectroscopic, eclipsing and resolved binaries. by

The first stringent condition follows from the maximal light deflection angle (12), given

$$
\varphi \leq 200 \mu \mathrm{as} \sqrt{\frac{M}{M_{\odot}} \frac{A}{\mathrm{AU}}} \frac{\mathrm{pc}}{r} .
$$

It represents a strict criterion for the maximal light deflection of a binary system with given Schwarzschild radius of component $\mathrm{A}$, given semi-major axis $A$, and given distance $r$ between binary system and the observer.

The second stringent condition on the orbital elements follows from the inclination formula in the simplified form as given by Eq. (19). For a better illustration, this condition is given in terms of angular degrees instead of radians. Using $\arctan x=x+\mathcal{O}\left(x^{3}\right)$ one obtains

$$
\left|90^{\circ}-i\right| \leq 2.25^{\circ} \frac{M}{M_{\odot}} \frac{\mu \text { as }}{\varphi} \frac{\mathrm{pc}}{r} .
$$

According to this strict condition, the inclination $i$ of a binary system with mass $M$ (recall $M$ is the stellar mass of component A) and at distance $r$ must not exceed the given value in order to have a light deflection $\varphi$.

Both these stringent conditions Eqs. (20) and (21) were already stated in the introductory Section by Eqs. (21) and (4), respectively. However, is should be underlined here, that both (20) and (21) were obtained with the aid of one and the same approach, namely the 
generalized lens equation, while (2) and (41) were obtained by means of the classical lens equation and the post-Newtonian solution, that means by two different approaches.

The observability of light deflection effect in binaries implies the realization of both these stringent conditions (20) and (21) simultaneously for a given binary system. But even if a given binary system satisfies these both conditions, the observability of light deflection effect is not guaranteed, because the astrometric position $E=0$ has to be reached during the time of observation. Nonetheless, as soon as the orbital elements $r, A, m$ and $i$ of the binaries are known, these both stringent conditions (201) and (21) allow to find a possible candidate for being a relevant binary system for a given light deflection $\varphi$ depending on the instrumentation of the observer. However, as it will be shown in Section $\nabla$ and Section VI. the existence of such systems is highly improbable.

\section{TOTAL NUMBER OF BINARIES WITH A GIVEN LIGHT DEFLECTION FOR INFINITE TIME OF OBSERVATION}

In the previous Section, the conditions on orbital parameters for a binary system have been determined in order to have a given magnitude of light deflection $\varphi$. In this Section, the total number of such relevant binaries is determined. In order to estimate the total number of binaries having a given light deflection $\varphi$, the following formula is applied:

$$
N(\varphi)=\int_{R_{\min }}^{R_{\max }} d^{3} r \rho(r) \int_{A_{\min }}^{A_{\max }} d A f(A) \int_{\mu_{\min }}^{\mu_{\max }} d \mu f(\mu) P(i) .
$$

Here, $\rho(r)$ is the density of binaries, $f(A)$ is the semi-major axis distribution of binary systems, $f(\mu)$ is the mass distribution of stars where $\mu=\frac{M}{M_{\odot}}$ is the mass-ratio of massive body (component A) and solar mass. The probability distribution $P(i)$ to find a binary system with given inclination $0 \leq i \leq \pi$, is a function of distance $r$, semi-major axis $A$, mass ratio $\mu$, and the given light deflection angle $\varphi$. According to (13), the probability distribution $P(i)$ is given by (the inclination of binary systems is of course a random distribution)

$$
P(i)=\frac{2}{\pi} \arccos \left(-\frac{p}{2}+\sqrt{\frac{p^{2}}{4}-q}\right),
$$

where $p$ and $q$ are given by Eqs. (14) and (15)).

For the minimal distance of a binary system from the Sun one can safely assume $R_{\text {min }}=$ 1 pc. From (12) follows that beyond a sphere with radius $R_{\max }=2000$ pc only a very few exceptional binary systems might have a light deflection of more than $1 \mu$ as. The Sun is located in the Orion arm which has about $1000 \mathrm{pc}$ across and approximately $3000 \mathrm{pc}$ in length. For the estimate according to (22), it is meaningful to assume that the stars are homogeneously distributed inside the Orion arm. For the uniform star density $\rho_{\text {stars }}=$ $0.1 \mathrm{star}_{\mathrm{pc}}^{-3}$, a value which is in line with the data of RECONS [2012]. Furthermore, a common presumption is, that about 50 percent of all stars are components of a binary or multiple system, see Duquennoy \& Mayor [1991] and Halbwachs, et al. [2003]. Then one obtains for the density of binaries

$$
\rho(r) \simeq 0.025 \text { binaries } \mathrm{pc}^{-3} .
$$


Let us now consider the distribution of semi-major axis $A$ in binary systems. Statistical investigations show that the distribution of binary semi-major axis is flat in a logarithmic scale over the range of six orders of magnitude, that means assumed to be valid in the large range $A_{\min }=10 R_{\odot} \leq A \leq 10^{4} \mathrm{AU}=A_{\max }$, see Kouwenhoven \& de Grijs [2008]. The lower limit $A_{\min }$ is determined by the semi-major axis at which Roche lobe overflow occurs, while the upper limit $\mathrm{A}_{\max }$ depends on how large the averaged star density is. The logarithmic distribution is known as "Öpik's law" (1924) named after its discoverer, and is given by $f(A) \sim \frac{1}{A}$, a law which has also been confirmed by recent investigations, see Poveda, et al. [2006]. This distribution is a consequence of the process of star formation as well as of the dynamical history of the binary system, and one can take this law as a given fact for numerical studies. Accordingly, see Appendix E:

$$
f(A)=\frac{1}{A}\left(\ln \frac{A_{\max }}{A_{\min }}\right)^{-1} .
$$

Furthermore, for the mass distribution $f(\mu)$ let us recall the initial mass function (IMF) which is the probablity that a star is newly formed with a stellar mass $M$ and is frequently assumed to be a power law $f(M) \sim M^{-\alpha}$. Originally, the IMF has been introduced by Salpeter [1955] for solar neighborhood region, who assumed the value $\alpha=2.35$ and a validity region for stars with masses between $0.4 M_{\odot}$ and $10 M_{\odot}$. During the past decades the IMF has been refined by several investigations. Especially, the numerical values of slope parameter $\alpha$ and regions of validity have been proposed in subsequent investigations, e.g. Scalo [1986], Robin, et al. [2003]. Ninkovic \& Trajkovska [2006], Ninkovic [1995], Kroupa [2001]; for a review see Kroupa [2002]. Moreover, the IMF does not necessarily coincide with the real mass distribution of stars, because IMF describes mass distribution of a star formation, while the solar neighborhood mainly consists of evolved stars of main sequence. Here, for simplicity this distribution is used as a given fact with $\alpha=2.35$, and the proposed region of validity $\mu_{\min }=0.4$, and $\mu_{\max }=10$ is assumed. According to IMF, one finds for $\alpha \neq 1$ (see Appendix E)

$$
f(\mu)=\frac{(1-\alpha) \mu^{-\alpha}}{\mu_{\max }^{(1-\alpha)}-\mu_{\min }^{(1-\alpha)}} .
$$

In order to motivate that distribution further, one can compare (26) with the RECONS data [2012] where one finds a fair agreement. Using (23) - (26), the results of the estimate (22) are shown in FIG. 4, recall, when evaluating this integral one has to take into account the boundary conditions given by Eqs. (11) and (12).

According to FIG. 4, in total there are about $N \sim 10^{3}$ binaries having a light deflection of at least $\varphi=1 \mu$ as. Here, the observation time is assumed to be infinite, so that it is guaranteed that the optimal configuration $E=0$ is reached.

\section{TOTAL NUMBER OF BINARIES WITH A GIVEN LIGHT DEFLECTION FOR FINITE TIME OF OBSERVATION}

In Eq. (22) the number of binaries with a given maximal possible light deflection $\varphi$ has been determined, just by taking for eccentric anomaly the value $E=0$, that means the ideal configuration where the light deflection takes its maximal value (note, the eccentricity 


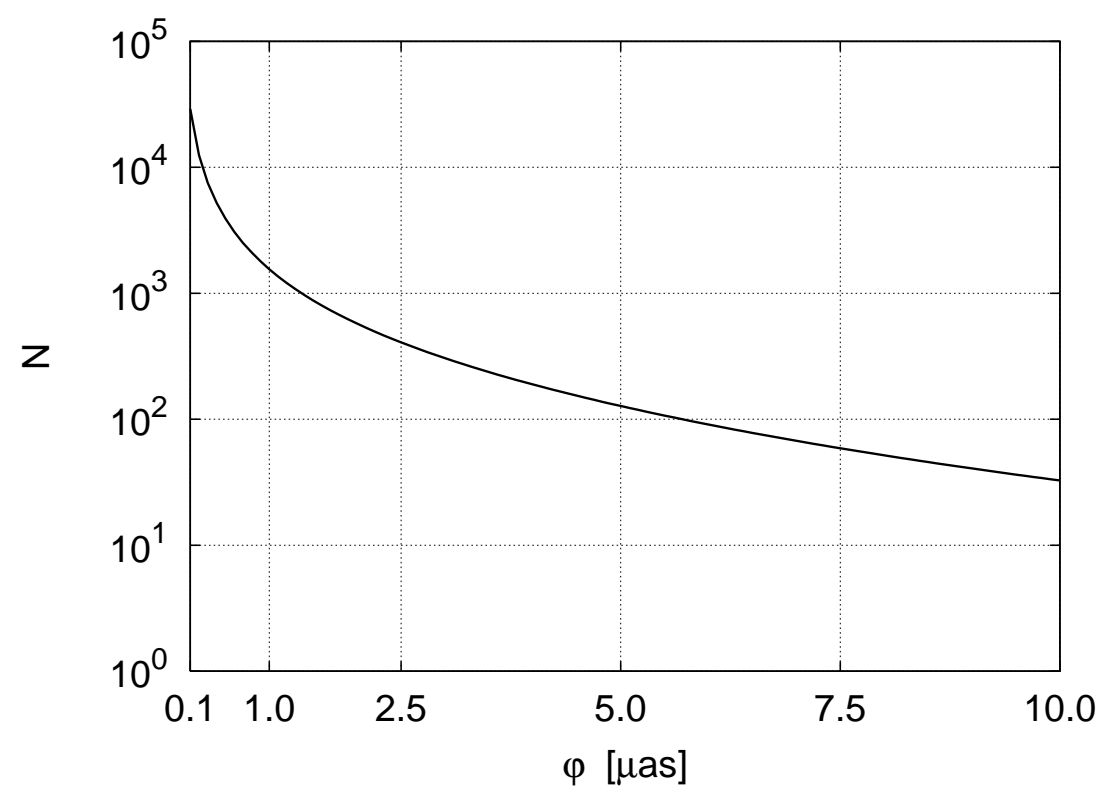

FIG. 4: Total number of binaries according to Eq. (22), having parameters such that the light deflection of component $\mathrm{B}$ at component $\mathrm{A}$ is larger than a given value for $\varphi$. Note, that for one individual observation the astrometric accuracy of Gaia is about $25 \mu$ as and the end-of-mission accuracy is about $5 \mu$ as in the most ideal case (bright star). The observation time is assumed to be infinite, so that it is guaranteed that the optimal configuration $E=0$ is reached.

$e=0)$. It is, however, obvious that during the most part of the orbital motion one will have $E \neq 0$ and the light deflection will be much smaller than the maximal possible light deflection angle $\varphi$. On the other side, the orbital period $T$ of relevant binaries, given by Eq. (A21), will easily exceed the time of observation; for instance, the Gaia mission time is about $T_{\text {mission }} \simeq 5$ years. Therefore, it will be not very probable, that the component $\mathrm{B}$ will be just at the relevant position near the value $E=0$, where the light deflection becomes observable on microarcsecond level. In order to determine that number of observable relevant binaries, one has to extend Eq. (22) as follows,

$$
N(\varphi)=\int_{R_{\min }}^{R_{\max }} d^{3} r \rho(r) \int_{A_{\min }}^{A_{\max }} d A f(A) \int_{\mu_{\min }}^{\mu_{\max }} d \mu f(\mu) P(i) P(E) .
$$

Here, $P(E)$ is the probability for the binary system to be in the region $E$, where the light deflection is larger than a given value for $\varphi$.

In the very same way, as applied for the derivation of the inclination formula (13), one can reconvert (B15) in terms of eccentric anomaly $E$ and one finds the eccentric anomaly formula:

$$
E= \pm \arccos \left(-\frac{p}{2}+\sqrt{\frac{p^{2}}{4}-q}\right)
$$

where $p$ and $q$ are given by Eqs. (14) and (15). For a given value of light deflection $\varphi$, the formula (28) yields the value of eccentric anomaly $E$ of a binary system characterized by semi-major axis $A$ and Schwarzschild radius (or mass) $m$ at a distance $r$. However, the 


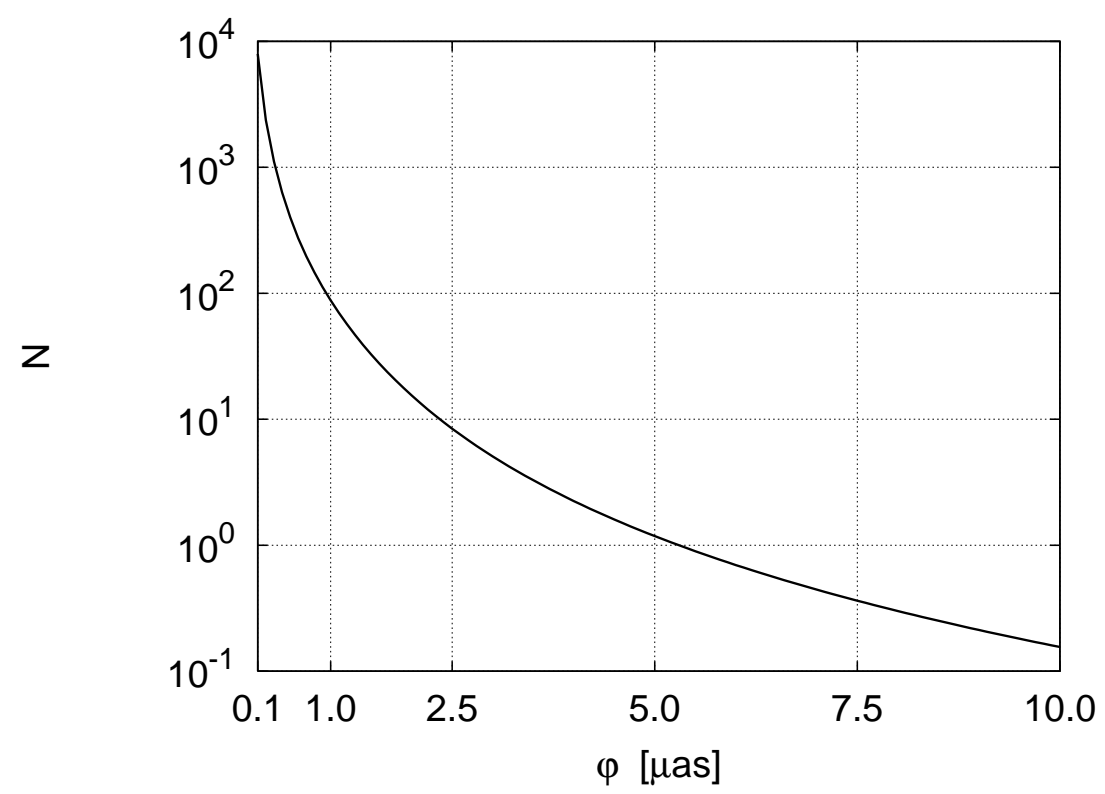

FIG. 5: Total number of binaries according to Eq. (27) where the binary system reaches the optimal configuration $E=0$ during an assumed observation time of $T_{\text {mission }}=5$ years, and having orbital parameters such that the light deflection of component $\mathrm{B}$ at component $\mathrm{A}$ is larger than a given value for $\varphi$. Note, that for one individual observation the astrometric accuracy of Gaia is about $25 \mu$ as and the end-of-mission accuracy is about $5 \mu$ as in the most ideal case (bright star).

values of $\varphi$ cannot be chosen arbitrarily, instead they are restricted by $\varphi_{\min }=\varphi\left(E= \pm \frac{\pi}{2}\right)$ and $\varphi_{\max }=\varphi(E=0)$ given by (of course, only astrometric positions with $0 \leq E \leq \frac{\pi}{2}$ are taken into account, because for the area $\frac{\pi}{2} \leq E \leq \pi$ the light deflection is negligible):

$$
\begin{aligned}
\varphi_{\min } & =\frac{1}{2}\left(\sqrt{\frac{A^{2}}{r^{2}}+8 \frac{m}{r} \frac{A}{r}}-\frac{A}{r}\right) \approx 2 \frac{m}{r}=0.0197 \mu \mathrm{as} \frac{M}{M_{\odot}} \frac{\mathrm{pc}}{r}, \\
\varphi_{\max } & =2 \frac{\sqrt{m A}}{r}=200 \mu \mathrm{as} \sqrt{\frac{M}{M_{\odot}} \frac{A}{\mathrm{AU}}} \frac{\mathrm{pc}}{r},
\end{aligned}
$$

where in (29) terms of the order $\mathcal{O}\left(\frac{m^{2}}{r A}\right)$ have been neglected. These expressions resemble the corresponding expressions in Eqs. (11) and (12). According to Eq. (28), the region where the binary system has a light deflection larger or equal $\varphi$ is given by $2 E$. One has also to take into account, that during Gaia mission time $T_{\text {mission }}$ the component $\mathrm{B}$ moves along the orbit and could move into the region $2 E$. Therefore, the probability $P(E)$ that the binary system is during Gaia mission time at least ones inside the relevant astrometric position with the value $E$ in (28), is given by

$$
P(E)=\mathcal{P}_{1}\left(\frac{1}{\pi} \arccos \left(-\frac{p}{2}+\sqrt{\frac{p^{2}}{4}-q}\right)+\frac{T_{\text {mission }}}{T}\right),
$$


where the operator $\mathcal{P}_{1}$ is defined by

$$
\mathcal{P}_{1}(x)=\begin{array}{lll}
x & \text { if } \quad x<1 \\
1 & \text { if } \quad x \geq 1
\end{array}
$$

The probability distribution (31) has to be implemented in Eq. (27) in order to determine the number of binary systems having a given light deflection $\varphi$ and to be observable during Gaia mission time $T_{\text {mission. }}$. The results of Eq. (27) are shown in FIG. 5, recall, when evaluating this integral one has to take into account the boundary conditions given by Eqs. (111) and (12). Accordingly, there are only a very few binaries $\sim 10^{2}$ having a light deflection of at least $\varphi=1 \mu \mathrm{as}$, if the astrometric position $E=0$ is reached during an assumed observation time of $T_{\text {mission }}=5$ years.

\section{SPECIAL CASE: CONDITIONS ON ORBITAL PARAMETERS FOR RESOLVED BINARIES}

In this Section, the special case of a resolved binary system is considered. In order to investigate the detectability of the light deflection effect in binaries, todays most modern astrometric mission, the ESA cornerstone mission Gaia, and its instrumentation which provides the highest possible astrometric accuracy at the moment, will be considered here as a concrete example.

\section{A. Resolving power of Gaia}

The core of Gaia optical instrumentation consists of two identical mirror telescopes, ASTRO-1 and ASTRO-2, with a rectangular pupil whose dimensions are $\mathrm{A}=0.50 \mathrm{~m}, \mathrm{~B}=$ $1.45 \mathrm{~m}$, and $f=35 \mathrm{~m}$ is the effective focal length. The intensity is given by, see Lattanzi, et al. [1998] and Lindgren [1998]:

$$
I\left(z_{\mathrm{A}}, z_{\mathrm{B}}\right)=I_{0}\left(\frac{\sin ^{2}\left(z_{\mathrm{A}}\right)}{z_{\mathrm{A}}^{2}} \frac{\sin ^{2}\left(z_{\mathrm{B}}\right)}{z_{\mathrm{B}}^{2}}\right),
$$

where $z_{\mathrm{A}}=\pi \mathrm{A} / \lambda \sin \Theta_{\mathrm{A}}, z_{\mathrm{B}}=\pi \mathrm{B} / \lambda \sin \Theta_{\mathrm{B}}, \mathrm{A}$ and $\mathrm{B}$ the width and length of rectangular mirror, $\lambda$ is the wavelength of incident light-ray, and $\Theta_{A}, \Theta_{B}$ are the angle of observation, i.e. the angle between the axis of the rectangular aperture and the line between aperture center and observation point. The intensity of incident light-ray at $\Theta_{A}=0, \Theta_{B}=0$ is denoted by $I_{0}$. The function $I\left(z_{\mathrm{A}}, z_{\mathrm{B}}\right) / I_{0}$ in Eq. (33) is the (by $\Theta_{\mathrm{A}}=\Theta_{\mathrm{B}}=0$ normalized) Point Spread Function (PSF) for monochromatic incident light with wavelength $\lambda$ for a rectangular aperture. The optical spectrum of stars is $\lambda=(350-750) \mathrm{nm}$. In FIG. 6 the PSF for an incident monochromatic light-ray with $\lambda=350 \mathrm{~nm}$ is represented for Gaia telescopes.

Most of the light is concentrated in the central bright rectangular shaped pattern. The length $l_{\mathrm{A}}$ and width $l_{\mathrm{B}}$ of this rectangle is determined by the first zero-roots of (33) at $z_{\mathrm{A}} \simeq \pi$ and $z_{\mathrm{B}} \simeq \pi$, respectively. From that it follows, that $\sin \Theta_{\mathrm{A}}=\pi \lambda /(\pi \mathrm{A})=\lambda / \mathrm{A}$, and $\sin \Theta_{\mathrm{B}}=\pi \lambda /(\pi \mathrm{B})=\lambda / \mathrm{B}$. Furthermore, if the diffraction pattern is shown on a screen at a distance $f$, then the length and width is given by, see Hog, et al. [1997]:

$$
L_{\mathrm{A}}=2 \frac{f \lambda}{\mathrm{A}}
$$




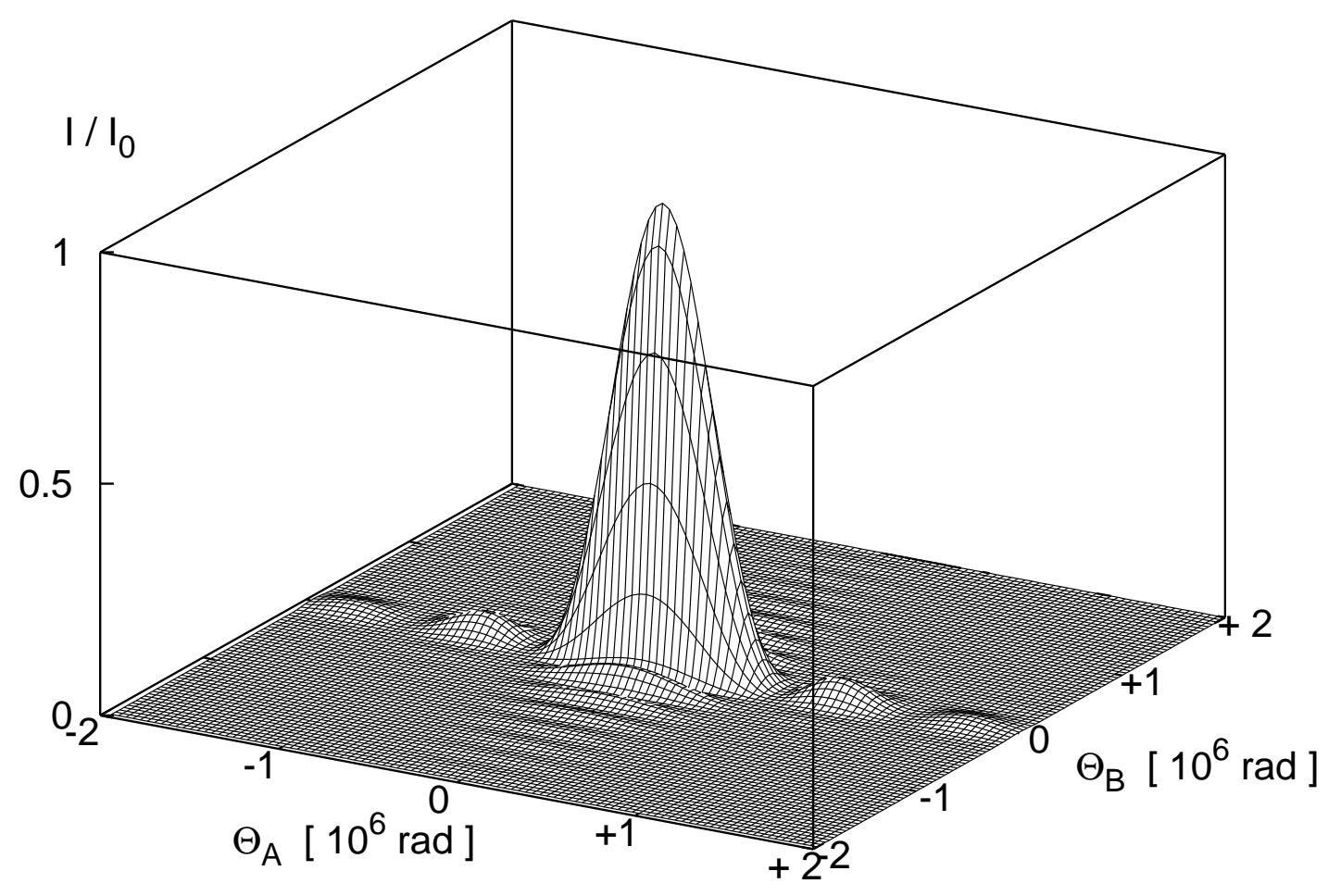

FIG. 6: Point Spread Function (PSF) for a rectangular telescope according to Eq. (33)). The incident monochromatic light-ray has a wavelength of $\lambda=350 \mathrm{~nm}$. The parameters of the rectangular telescope are: $\mathrm{A}=0.5 \mathrm{~m}, \mathrm{~B}=1.45 \mathrm{~m}$.

$$
L_{\mathrm{B}}=2 \frac{f \lambda}{\mathrm{B}}
$$

where $f$ is the focal length of the optic, i.e. of the rectangular Gaia mirror. Accordingly, the given numerical values $\mathrm{A}=0.50 \mathrm{~m}, \mathrm{~B}=1.45 \mathrm{~m}$, and $\lambda=350 \mathrm{~nm}$ result in $L_{\mathrm{A}}=49.0 \mu \mathrm{m}$ and $L_{\mathrm{B}}=16.9 \mu \mathrm{m}$ for the length and width of "Airy rectangle" of Gaia optics. Note, that the "Airy rectangle" has the same order of magnitude than the pixel size $(10 \mu \mathrm{m} \times 30 \mu \mathrm{m})$ of the 110 CCDs (Charge-Coupled Device) sensors of astrometric field part of the focal plane. In order to separate two pointlike sources, the distance between their centers of the rectangle has to be larger than either $L_{\mathrm{A}}$ or $L_{\mathrm{B}}$. Since $L_{\mathrm{B}}<L_{\mathrm{A}}$, in this study the better resolution value $L_{\mathrm{B}}$ is used, which corresponds to a resolution angle of

$$
\delta=\frac{L_{\mathrm{B}}}{2 f}=\frac{\lambda}{\mathrm{B}} .
$$

The resolving power is the minimal angular distance between two objects to get separable by Gaia instrumentation. With the parameters given above one obtains the resolving power $\delta$ of Gaia optics:

$$
\delta=0.24 \times 10^{-6} \mathrm{rad}=49.7 \text { mas } .
$$


In what follows this parameter is of fundamental importance in order to determine the ability of Gaia to determine the light deflection in binary systems.

\section{B. Orbital Parameters of Resolved Binaries Observable by Gaia}

In this Section, the question is addressed which and how many binary systems can be separated by Gaia instrumentation among all those relevant binaries found in the previous Section; see FIG. 5. In average, Gaia will observe each object 80 times, but will not constantly observe these objects during mission time. However, for simplicity the scanning law of Gaia is approximated by assuming a permanent observation of all objects during the whole mission time.

Furthermore, visual binaries are considered in this Section, i.e. binaries which are separable by Gaia telescopes. The both largest telescopes of Gaia have a resolution angle $\delta$ discussed in the previous Section, see Eqs. (36) and (37). For binary systems, this resolution angle $\delta$ corresponds to a minimal distance between the components A and B to get separable within Gaia optics. Using ( $(\underline{B 6})$ and (36) one obtains the condition

$$
d=A|\cos i| \geq \delta r=\frac{\lambda}{B} r
$$

where $r$ is the distance between the remote objects and Gaia observer. This condition is by far much more important than taking into account the effect of finite radius of the stars, which would imply $A|\cos i| \geq R_{A}$, where $R_{A}$ being the radius of component $A$. By inserting the extreme case $A|\cos i|=\delta r$ into (10), one obtains

$$
\varphi=\frac{1}{2}\left(\sqrt{\delta^{2}+8(1+\sin i) \frac{m}{r} \frac{A}{r}}-\delta\right) \simeq 4 \frac{m}{r} \frac{A}{r} \frac{1}{\delta},
$$

where $\sin i \simeq 1$ has been used, and terms of higher order $\mathcal{O}\left(\mathrm{m}^{2}\right)$ are neglected. Relation (39) is an expression for the maximal light deflection angle of a binary system when taking into account the resolving power of Gaia. Eq. (39) is a much stricter restriction than the generalized lens equation (10), because (39) determines the light deflection angle only of those binary systems having a resolution angle $\delta$ of Gaia optical instrumentation, while (10) determines the light deflection angle of any possible binary system. Note, that from Eq. (39) follows the maximal possible distance of visual binaries for a given light deflection:

$$
r \leq \sqrt{4 \frac{m A}{\varphi \delta}}=0.18 \mathrm{pc} \sqrt{\frac{M}{M_{\odot}} \frac{A}{\mathrm{AU}}},
$$

where in the last expression the optimal values $\delta=0.24 \times 10^{-6} \mathrm{rad}$ and $\varphi=25 \mu$ as has been used. The condition (40) can also be written by

$$
A \geq 30 \mathrm{AU} \frac{M_{\odot}}{M} \frac{r^{2}}{\mathrm{pc}^{2}} .
$$

These both conditions (40) and (41) imply rather extreme orbital parameters on visual binaries. For instance, condition (40) implies a maximal distance of $r \leq 18 \mathrm{pc}$ for solar-mass type binaries even with a huge semi-major axis of $A=10^{4} \mathrm{AU}$, while condition (41) implies a large semi-major axis for solar-mass type binaries outside a sphere of $r \geq 10 \mathrm{pc}$. It is almost certain, that such extreme parameters will not be realized in nature. 


\section{SUMMARY}

In this study, the light deflection in binary systems has been considered. While there is absolutely not any doubt about the existence of this relativistic effect, is has not been observed so far. To investigate this effect of light deflection, an inclination formula (13) has been derived by means of generalized lens equation (9) obtained recently by Zschocke [2011], and these both equations are the theoretical basis for investigating the light deflection effect in binary systems. A simplified inclination formula has been presented by Eq. (19) and its validity has been discussed in some detail. This simplified inclination formula has also been obtained by Klioner, et al. [2003] by an independent approach. Furthermore, two stringent conditions on the orbital parameters have been given by Eqs. (20) and (21). These both stringent conditions are relations between the orbital elements of a (resolved, astrometric, eclipsing, spectroscopic) binary system for a given magnitude of light deflection, and allow to find a relevant binary system in a straightforward way.

In Section V, the total number of binaries with a given light deflection has been determined by means of the semi-major axis distribution according to "Öpik's law" and the mass distribution according to "Salpeter's mass distribution". Since the inclinations are randomly distributed, the inclination formula allows to estimate the total number of relevant binaries with the aid of Eq. (22). It turns out, that in total there exist about $10^{3}$ binaries having orbital parameters such that the light deflection amounts to be at least $1 \mu$ as; see FIG. 4 .

In Section VI a finite time of observation of 5 years (Gaia mission time) has been considered, which considerably reduces the total number of relevant binaries. Clearly, this case is of more practical importance, since a restricted time window of observation is in more agreement with reality than the first scenario. By taking into account the probability to find the system in the ideal astrometric position $E=0$ where the light deflection becomes maximal, it has been found by evaluating the corresponding integral (27) that there is not any of the relevant binary systems in the ideal position $E=0$ during Gaia mission time; see FIG. 5. Thus, while in principle a few binaries will have a significant light deflection, the effect could not be detected due to the restricted finite time window of observation.

Furthermore, the special case of resolved binaries has been considered in Section VII. The astrometric instrumentation of the ESA cornerstone mission Gaia, see e.g. Perryman, et al. [2001], has been considered in some detail in order to decide whether or not this subtle effect of light deflection can be observed. Two conditions for resolved binaries were presented in Eqs. (40) and (41) for such special kind of binary systems. It has been shown, however, that even for the Gaia mission, which is an outstanding milestone of progress in astrometry, such binary systems must have rather extreme orbital parameters in order to reach todays level of detectability, i.e. on microarcsecond level. The existence of such exotic binaries is, however, highly improbable.

In summary, the main results are presented by the inclination formulae (13) and its simplified version (19), the stringent conditions (20) and (21) and by the diagrams FIG. 4 and FIG. 5. Accordingly, one comes to the conclusion that the detectability of light deflection in binary systems reaches the technical limit of todays high precision astrometry and might be detected only in case of a very few and highly exotic binary systems. It is, however, very unlikely that such extreme binaries might exist. It seems that the detection of the light deflection effect in binary systems needs an astrometric accuracy of better than about $0.1 \mu$ as. Thus, only astrometric missions of the next generation can accept the challenge to detect this subtle effect of relativity. 


\section{Acknowledgements}

This work was partially supported by the BMWi grants 50 QG 0601 and 50 QG 0901 awarded by the Deutsche Zentrum für Luft- und Raumfahrt e.V. (DLR). Enlighting discussions with Prof. Sergei A. Klioner, Prof. Michael H. Soffel and Prof. Francois Mignard are gratefully acknowledged.

[2008] Bozza, V., 2008, Phys. Rev. D, 78, 103005

[1991] Brumberg, V. A. 1991, Essential Relativistic Celestial Mechanics, (Bristol: Adam Hilder) [1983] Chandrasekhar, S., 1983, The Mathematical Theory of Black Holes (Oxford: Clarendon) [1991] Duquennoy, A. \& Mayor, M., 1991, A\&A, 248, 485

[2000] Fritelli, S., Kling, T.P. \& Newmann, E.T., 2000, Phys. Rev. D, 64, 064021

[2003] Halbwachs, J.L., Mayer, M., Udry, S. \& Arenou, F., 2003, A\&A, 397, 159

[1997] Hog, E., Bastian, U. \& Seifert, W., 1997, Optical Design of Gaia, www.en.scientificcommons.org

[2003] Klioner, S.A., 2003, AJ 125, 1580

[2003] Klioner, S.A., Mignard, F. \& Soffel, M., 2003, Astrometric Signature of Gravitational Microlensing on the Components of Edge-On Binary Systems, unpublished

[2010] Klioner, S.A. \& Zschocke, S., 2010 Class. Quantum Grav., 27, 075015

[2008] Kouwenhoven, M.B.N. \& de Grijs, R., 2008, A\&A, 480, 103

[2001] Kroupa, P., 2001, MNRAS, 322, 231

[2002] Kroupa, P., 2002, Science, 295, 82.

[1976] Landau, L.D. \& Lifshitz, E.M., 1976, Mechanics, Vol. 1, (Oxford Pergamon Press)

[1998] Lattanzi, M., Carollo, D. \& Gai, M., 1998, Aberrated point spread function's for the MMS configuration and astrometric error, Technical Report, SAG-ML-014; available at the Gaia document archive http://www.rssd.esa.int/llink/livelink.

[1998] Lindgren, L., 1998, Point spread functions for Gaia including aberrations, Technical Report from Lund Observatory, SAG-LL-025; available at the Gaia document archive http://www.rssd.esa.int/llink/livelink

[1995] Ninkovic, S., 1995, Bull. Astron. Belgrade, 151, 1

[2006] Ninkovic, S. \& Trajkovska, V., 2006, Serb. AJ, 172, 17

[1924] Öpik, E.J., 1924, Tartu Obs. Publ., 25

[2001] Perryman, M.A.C., de Boer, K., Gilmore, G. et al., A\&A, 2001, 369, 339

[2006] Poveda, A., Allen, C. \& Hernandez-Alcantara, A., 2007, in Proc. IAU Symp. 240; arXiv: astro-ph/0705.2021

[2012] RECONS, 2012, http://www.recons.org/

[2003] Robin, A.C., Reyle, C., Derriere S. \& Picaud, S., 2003, A\&A, 409, 523

[1955] Salpeter, E.E., 1955, AJ, 121, 161

[1986] Scalo, J.M., 1986, Fundam. Cosm. Phys., 11, 1

[2011] WDS, 2011, http://ad.usno.navy.mil/wds/wds.html

[2011] Zschocke, S. 2011, A generalized lens equation for light deflection in weak gravitational fields, Class. Quantum Grav. 28, 125016

[2004] Zwitter, T. \& Munari, U., 2004, The Environment and Evolution of Double and Multiple Stars, RevMexAA (SC), 21, 25 


\section{Appendix A: Two-body problem}

The calculations in this Appendix follow mainly Landau \& Lifshitz [1976]. Consider two massive bodies, one component having a mass $M_{A}$ and spatial coordinate $\boldsymbol{r}_{A}$, and second component with a mass $M_{B}$ and spatial coordinate $\boldsymbol{r}_{B}$, respectively. They orbit around their common center of mass $\boldsymbol{r}_{\mathrm{CMS}}$,

$$
\boldsymbol{r}_{\mathrm{CMS}}=\frac{1}{M_{A}+M_{B}}\left(M_{A} \boldsymbol{r}_{A}+M_{B} \boldsymbol{r}_{B}\right)
$$

The Lagrangian $\mathcal{L}$ of the two-body problem is given by

$$
\mathcal{L}=\frac{M_{A}}{2} \dot{\boldsymbol{r}}_{A}^{2}+\frac{M_{B}}{2} \dot{\boldsymbol{r}}_{B}^{2}-U\left(\left|\boldsymbol{r}_{A}-\boldsymbol{r}_{B}\right|\right)
$$

where $U$ is the potential. With the aid of relative coordinate $\boldsymbol{r}_{A B}=\boldsymbol{r}_{A}-\boldsymbol{r}_{B}$ and reduced mass $\bar{M}=M_{A} M_{B} /\left(M_{A}+M_{B}\right)$, the two-body problem can be transformed into into onebody problem,

$$
\mathcal{L}=\frac{\bar{M}}{2} \dot{\boldsymbol{r}}_{A B}^{2}-U\left(r_{A B}\right) .
$$

Polar coordinates $\left(r_{A B}, \phi\right)$ yield

$$
\mathcal{L}=\frac{1}{2}\left(\bar{M} \dot{r}_{A B}^{2}+r_{A B}^{2} \dot{\phi}^{2}\right)-U\left(r_{A B}\right) .
$$

The orbital angular momentum $L$ is conserved

$$
L=\bar{M} r_{A B}^{2} \dot{\phi}=\text { const },
$$

by means of which one obtains for the total energy of the two-body system the expression

$$
E=\frac{\bar{M}}{2} \dot{r}_{A B}^{2}+\frac{L^{2}}{2 \bar{M} r_{A B}^{2}}+U\left(r_{A B}\right) .
$$

From Eq. (A6) one deduces

$$
\dot{r}_{A B}=\left(\frac{2}{\bar{M}}\left[E-U\left(r_{A B}\right)\right]-\frac{L^{2}}{\bar{M}^{2} r_{A B}^{2}}\right),
$$

and from Eq. (A7) one obtains up to an integration constant

$$
\begin{aligned}
t & =\int d r_{A B}\left(\frac{2}{\bar{M}}\left[E-U\left(r_{A B}\right)\right]-\frac{L^{2}}{\bar{M}^{2} r_{A B}^{2}}\right)^{-1 / 2}, \\
\phi & =\int d r_{A B} \frac{\bar{M}}{r_{A B}^{2}}\left(2 \bar{M}\left[E-U\left(r_{A B}\right)\right]-\frac{L^{2}}{r_{A B}^{2}}\right)^{-1 / 2},
\end{aligned}
$$

where in the second relation Eq. (A5) has been used; note that (A9) is the relation between $r_{A B}$ and $\phi$ and is called orbital equation. The Eqs. (A8) and (A9) are the general integral 
solutions of a two-body problem. In order to integrate these Eqs. (A8) and (A9) one has to specify the potential $U$. In case of Kepler problem one has

$$
U(r)=-\frac{\alpha}{r_{A B}} \quad \text { with } \quad \alpha=G M_{A} M_{B} .
$$

The Eq. (A9) can be integrated and yields

$$
\phi=\arccos \left(\frac{L}{r_{A B}}-\frac{\gamma \bar{M} M_{A} M_{B}}{L}\right)\left(2 \bar{M} E+\frac{\gamma^{2} \bar{M}^{2} M_{A}^{2} M_{B}^{2}}{L^{2}}\right)^{-1 / 2},
$$

where the axes are chosen such that the mentioned integration constant vanishes. Furthermore, by introducing the eccentricity e (possible values of eccentricity are between $0 \leq \mathrm{e}<1$; $\mathrm{e}=0$ corresponds to a circular orbit),

$$
\mathrm{e}=\left(1+\frac{2 E L^{2}\left(M_{A}+M_{B}\right)}{\gamma^{2} M_{A}^{3} M_{B}^{3}}\right)^{1 / 2}
$$

the solution (A11) can be written as

$$
\frac{1}{r_{A B}} \frac{L^{2}}{\gamma \bar{M} M_{A} M_{B}}=1+\mathrm{e} \cos \phi
$$

Note the expressions of semi-major axis $A$ and semi-minor axis $B$,

$$
\begin{aligned}
A & =\frac{L^{2}}{\left(1-\mathrm{e}^{2}\right) \gamma \bar{M} M_{A} M_{B}}, \\
B & =\frac{L^{2}}{\sqrt{1-\mathrm{e}^{2}} \gamma \bar{M} M_{A} M_{B}} .
\end{aligned}
$$

To solve the integral (A8), one substitutes

$$
r_{A B}-A=-A \text { e } \cos E,
$$

where $E$ is called eccentric anomaly. Then, one obtains for the integral in Eq. (A8) the expression

$$
t=\left(\frac{A^{3}}{\gamma\left(M_{A}+M_{B}\right)}\right)^{1 / 2} \int d E(1-\mathrm{e} \cos E),
$$

and the solution is given by

$$
t=\left(\frac{A^{3}}{\gamma\left(M_{A}+M_{B}\right)}\right)^{1 / 2}(E-\mathrm{e} \sin E)
$$

where the integration constant vanishes, i.e. the particle at $t=0$ is in periastron. The Eqs. (A13) and (A18) are the general solutions of two-body problem. They can be rewritten as

$$
\begin{aligned}
r_{A B} & =A(1-\mathrm{e} \cos E) \\
t & =\left(\frac{A^{3}}{\gamma\left(M_{A}+M_{B}\right)}\right)^{1 / 2}(E-\mathrm{e} \sin E) .
\end{aligned}
$$


In case of ellipse, $E=0$ in periastron, $E=\pi$ in apastron, and for a complete orbit $E$ runs from $E=0$ to $E=2 \pi$. Thus, one obtains for the orbital period the expression

$$
T=2 \pi\left(\frac{A^{3}}{\gamma\left(M_{A}+M_{B}\right)}\right)^{1 / 2} .
$$

Note, the solution $\boldsymbol{r}$ in Cartesian coordinates, $x=r_{A B} \cos \phi$ and $y=r_{A B} \sin \phi$ :

$$
\begin{aligned}
\boldsymbol{r}_{A B} & =\left(\begin{array}{l}
x \\
y
\end{array}\right), \\
x & =A(\cos E-\mathrm{e}), \\
y & =A\left(1-\mathrm{e}^{2}\right)^{1 / 2} \sin E .
\end{aligned}
$$

The coordinates of the bodies A and B, i.e. their orbits, are given by

$$
\begin{aligned}
& \boldsymbol{r}_{A}=\boldsymbol{r}_{\mathrm{CMS}}+\frac{\boldsymbol{r}_{A B}}{1+\frac{M_{A}}{M_{B}}}, \\
& \boldsymbol{r}_{B}=\boldsymbol{r}_{\mathrm{CMS}}-\frac{\boldsymbol{r}_{A B}}{1+\frac{M_{B}}{M_{A}}} .
\end{aligned}
$$

Accordingly, the geometry of the orbit is determined by two orbital parameters: semi-major axis $\mathrm{A}$ and eccentricity e. In order to know the position of one celestial body, either component $\mathrm{A}$ or component $\mathrm{B}$, two additional orbital parameters are needed, namely orbital period $T$ and true anomaly $\nu$. A geometrical representation of the coordinates of the components of a binary star is given in FIG. 7 for the case of $M_{A}=1.5 M_{\odot}, M_{B}=1.0 M_{\odot}, \mathrm{e}=0.5, A=$ $2 \mathrm{AU}$.

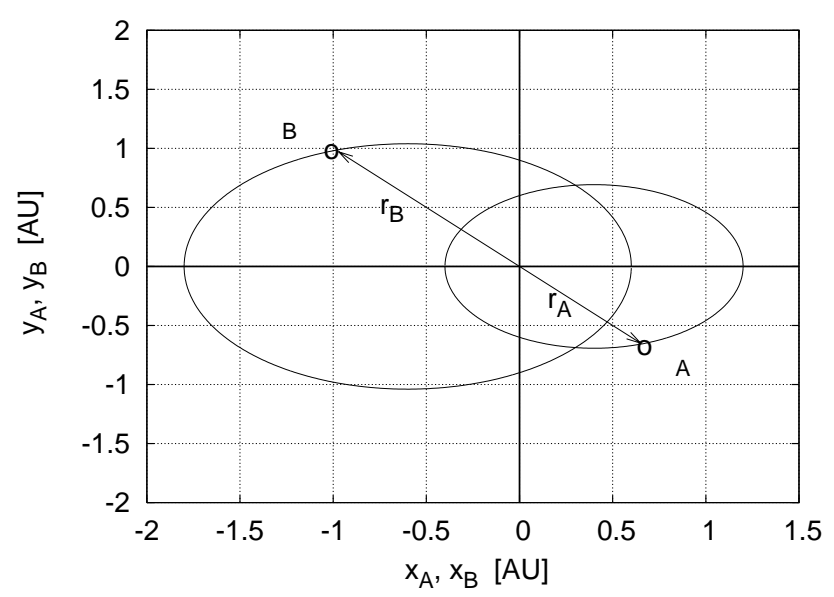

FIG. 7: Geometrical representation of the coordinates of a binary star. In the example considered, the masses are $M_{A}=1.5 M_{\odot}$ and $M_{B}=1.0 M_{\odot}$, respectively. The semi-major axis of the binary system is chosen $\mathrm{A}=2 \mathrm{AU}$ and eccentricity is taken $\mathrm{e}=0.5$. The coordinates of mass center are $\boldsymbol{r}_{\mathrm{CMS}}=\mathbf{0}$. The massive bodies A and B are always in opposition to each other. 


\section{Appendix B: Derivation of Eq. (10)}

For the inclination formula the impact of eccentricity on light deflection is neglected, thus $e=0$, implying that $\omega=0$ is taken. Then, for the vectors from massive body to observer $\boldsymbol{x}_{1}$ and from massive body to source $\boldsymbol{x}_{0}$, one has

$$
\begin{gathered}
\boldsymbol{x}_{1}=r\left(\begin{array}{c}
\sin i-\epsilon_{1} \cos E \\
-\epsilon_{1} \sin E \\
\cos i
\end{array}\right) \\
\boldsymbol{x}_{0}=-A\left(\begin{array}{c}
\cos E \\
\sin E \\
0
\end{array}\right)
\end{gathered}
$$

where the small parameter

$$
\epsilon_{1}=\frac{A}{r} \frac{m_{B}}{m_{A}+m_{B}} \ll 1
$$

has been introduced. From Eqs. (BB1) and (BB2) one obtains for vector $\boldsymbol{k}=\boldsymbol{R} / R$, where $\boldsymbol{R}=\boldsymbol{x}_{1}-\boldsymbol{x}_{0}$, the expression

$$
\boldsymbol{k}=\frac{1}{\sqrt{1+2 \epsilon_{2} \sin i \cos E+\epsilon_{2}^{2}}}\left(\begin{array}{c}
\sin i+\epsilon_{2} \cos E \\
\epsilon_{2} \sin E \\
\cos i
\end{array}\right)
$$

where the small parameter

$$
\epsilon_{2}=\frac{A}{r} \frac{m_{A}}{m_{A}+m_{B}} \ll 1
$$

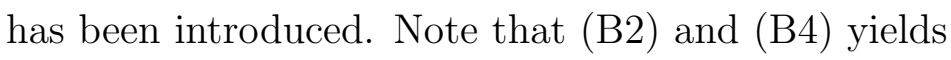

$$
d=\left|\boldsymbol{k} \times \boldsymbol{x}_{0}\right|=A|\cos i|\left(1+\mathcal{O}\left(\epsilon_{2}\right)\right) .
$$

Using (B1) - (B5), the generalized lens equation (91) reads

$$
\varphi=\frac{1}{2} \frac{1}{T_{1} T_{2}}\left(\sqrt{W_{1}^{2}+8 \frac{m}{r} \frac{A}{r}\left(T_{0}+T_{1}-\epsilon_{1}\right) T_{2}}-W_{1}\right),
$$

where $W_{1}=\frac{A}{r} \sqrt{1-T_{0}^{2}}$ and

$$
\begin{aligned}
& T_{0}=\sin i \cos E \\
& T_{1}=\sqrt{1-2 \epsilon_{1} \sin i \cos E+\epsilon_{1}^{2}}, \\
& T_{2}=\sqrt{1+2 \epsilon_{2} \sin i \cos E+\epsilon_{2}^{2}} .
\end{aligned}
$$


By series expansion one obtains up to terms of order $\mathcal{O}\left(\frac{A}{r} \sqrt{\frac{m}{r}} \frac{A}{r}\right)$ :

$$
\varphi=\frac{1}{2}\left(\sqrt{W_{2}^{2}+8 \frac{m}{r} \frac{A}{r}(1+w)}-W_{2}\right)
$$

where $W_{2}=\frac{A}{r} \sqrt{1-w^{2}}$ with $w=\sin i \cos E$. The minimal and maximal light deflection angle are

$$
\begin{aligned}
& \varphi_{\min }=\varphi\left(i=\frac{\pi}{2}, E=\pi\right)=0 \\
& \varphi_{\max }=\varphi\left(i=\frac{\pi}{2}, E=0\right)=2 \frac{\sqrt{m A}}{r} .
\end{aligned}
$$

In this study, the maximal possible light deflection effect is of interest. Accordingly, two configurations are relevant:

$$
\varphi(E=0)=\frac{1}{2}\left(\sqrt{\frac{A^{2}}{r^{2}} \cos ^{2} i+8 \frac{m}{r} \frac{A}{r}(1+\sin i)}-\frac{A}{r}|\cos i|\right),
$$

up to order $\mathcal{O}\left(\frac{A}{r} \sqrt{\frac{m}{r} \frac{A}{r}}\right)$ which is just Eq. (10), and

$$
\varphi\left(i=\frac{\pi}{2}\right)=\frac{1}{2}\left(\sqrt{\frac{A^{2}}{r^{2}} \sin ^{2} E+8 \frac{m}{r} \frac{A}{r}(1+\cos E)}-\frac{A}{r}|\sin E|\right),
$$

up to order $\mathcal{O}\left(\frac{A}{r} \sqrt{\frac{m}{r} \frac{A}{r}}\right)$. Furthermore, it is useful to take into account only astrometric positions with $0 \leq E \leq \frac{\pi}{2}$, because otherwise the light deflection is for sure negligible.

\section{Appendix C: Derivation of Eq. (13)}

From (B14) one obtains

$$
\left(2 \varphi+\frac{A}{r}|\cos i|\right)^{2}=\frac{A^{2}}{r^{2}} \cos ^{2} i+8 \frac{m}{r} \frac{A}{r}(1+\sin i) .
$$

From (C1) one obtains

$$
\begin{aligned}
& \left(\varphi^{2}+4 \frac{m^{2}}{r^{2}}\right) \frac{A^{2}}{r^{2}} \sin ^{2} i+4 \frac{m}{r} \frac{A}{r}\left(2 \frac{m}{r} \frac{A}{r}-\varphi^{2}\right) \sin i \\
= & \left(\frac{A^{2}}{r^{2}}+4 \frac{m}{r} \frac{A}{r}\right) \varphi^{2}-4 \frac{m^{2}}{r^{2}} \frac{A^{2}}{r^{2}}-\varphi^{4} .
\end{aligned}
$$


Eq. (C2) represents an quadratic equation for the expression $|\sin i|$, which has the following both solutions for the inclination $i$ :

$$
\sin i=\left(-\frac{p}{2} \pm \sqrt{\frac{p^{2}}{4}-q}\right)
$$

where

$$
\begin{aligned}
& p=\frac{8 m^{2} A-4 m r^{2} \varphi^{2}}{A\left(r^{2} \varphi^{2}+4 m^{2}\right)} \\
& q=-\frac{A^{2} r^{2} \varphi^{2}+4 m A r^{2} \varphi^{2}-4 m^{2} A^{2}-r^{4} \varphi^{4}}{A^{2}\left(r^{2} \varphi^{2}+4 m^{2}\right)}
\end{aligned}
$$

Eq. (C3) represents two solutions, however only the one with the plus-sign is valid. This can be shown as follows. For the value $i=\frac{\pi}{2}$ the light deflection has to be $\varphi=\varphi_{\max }=2 \sqrt{m A} / r$, according to (12). By inserting $\varphi_{\max }$ in Eqs. (C4) and (C5) one obtains $p=-2 \mathrm{~m} /(A+m)$ and $q=-(A-m) /(A+m)$. If one inserts $i=\frac{\pi}{2}$ for $p$ and $q$ into Eq. (C3) one obtains the relation

$$
1=\frac{m}{A+m} \pm \sqrt{\frac{m^{2}}{(A+m)^{2}}+\frac{A-m}{A+m}}=\frac{m}{A+m} \pm \frac{A}{A+m} .
$$

Obviously, relation (C6) is only correct for the upper sign. A very similar proof can also be done using $\varphi_{\text {min }}$ which also yields that the upper sign is the correct and only solution. Therefore, the inclination formula is given by (note, that in the region under consideration $\sin i=\sin (\pi-i))$

$$
i=\frac{\arcsin \left(-\frac{p}{2}+\sqrt{\frac{p^{2}}{4}-q}\right) \quad \text { for } \quad 0 \leq i \leq \frac{\pi}{2},}{\pi-\arcsin \left(-\frac{p}{2}+\sqrt{\frac{p^{2}}{4}-q}\right) \quad \text { for } \quad \frac{\pi}{2}<i \leq \pi .}
$$

For the complete region $0 \leq i \leq \pi$ one obtains for the inclination formula the following expression:

$$
\left|\frac{\pi}{2}-i\right|=\arccos \left(-\frac{p}{2}+\sqrt{\frac{p^{2}}{4}-q}\right),
$$

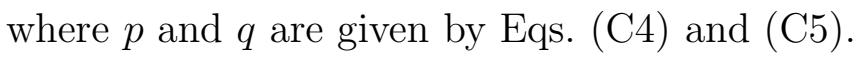

\section{Appendix D: Derivation of Eq. (3)}

In this appendix, some basic steps of the calculations of Klioner, et al. [2003] are presented. A scheme of the light propagation in a binary system is presented by Fig. 2, The light signal from component B, considered to be the light source, is deflected by component 
A, considered to be the massive body which curves the space-time. The vector $\boldsymbol{x}_{1}$ points from the mass center of massive body to the observer, and vector $\boldsymbol{x}_{0}$ points from the mass center of massive body to the source; $\boldsymbol{R}=\boldsymbol{x}_{1}-\boldsymbol{x}_{0}$ and its absolute value $R=|\boldsymbol{R}|$, and $m=\frac{G M}{c^{2}}$ is the Schwarzschild radius of massive body; the explicit label A is omitted. Furthermore we define the impact vector $\boldsymbol{d}=\boldsymbol{k} \times\left(\boldsymbol{x}_{1} \times \boldsymbol{k}\right)$ is defined, and its absolute value $d=|\boldsymbol{d}|$, see also FIG. 2.

According to Klioner \& Zschocke [2010], the transformation of $\boldsymbol{k}$ to the unit tangent vector $\boldsymbol{n}$ of light-trajectory at observer is in standard post-Newtonian approach given by

$$
\boldsymbol{n}=\boldsymbol{k}-2 m \frac{\boldsymbol{k} \times\left(\boldsymbol{x}_{0} \times \boldsymbol{x}_{1}\right)}{x_{1}\left(x_{0} x_{1}+\boldsymbol{x}_{0} \cdot \boldsymbol{x}_{1}\right)}+\mathcal{O}\left(m^{2}\right) .
$$

Note, the PPN parameter of parameterized post-Newtonian formalism, which characterizes a possible deviation of the physical reality from general theory of gravity, is set equal to 1 here for simplicity. This expression is valid as long as $d \gg m$, but diverges for $d \rightarrow 0$. Thus, it is not valid for all possible binary configurations, instead one has take care to consider only such astrometric configurations with $d \gg m$. By means of (D1) one obtains for the light deflection angle $\varphi$, i.e. for the angle between $\boldsymbol{n}$ and $\boldsymbol{k}$, the expression

$$
\varphi=2 \frac{m}{r} \tan \frac{\psi}{2}
$$

where $\frac{\sin \psi}{1+\cos \psi}=\tan \frac{\psi}{2}, x_{1}=r+\mathcal{O}(A)$ has been used, and $\psi$ is the angle between $\boldsymbol{x}_{0}$ and $\boldsymbol{x}_{1}$. The expression (D2) diverges for $\psi \rightarrow \pi$, which corresponds with the mentioned divergence of (D1) for $d \rightarrow 0$. Obviously, $\psi \leq i+\frac{\pi}{2}$ (from Eq. (28) it is clear that eccentric anomaly $E$ of binary system should be very close to zero for the light deflection effect to be observable at the level of microarcsecond, i.e. one actually could even assume $\psi \simeq i+\frac{\pi}{2}$ ) and one obtains

$$
\varphi \leq 2 \frac{m}{r} \tan \left(\frac{i}{2}+\frac{\pi}{4}\right)=2 \frac{m}{r} \cot \left(\frac{\pi}{4}-\frac{i}{2}\right)
$$

where $\tan \left(\alpha+\frac{\pi}{2}\right)=-\cot \alpha$ has been used, $\cot \alpha=\tan ^{-1} \alpha$, and the asymmetry of function $\cot \alpha$. From (D3) one obtains

$$
\left|\frac{\pi}{2}-i\right|_{\mathrm{KMS}} \leq 2 \arctan \left(0.0197 \frac{M}{M_{\odot}} \frac{\mu \mathrm{as}}{\varphi} \frac{\mathrm{pc}}{r}\right),
$$

where $\frac{m}{m_{\odot}}=\frac{M}{M_{\odot}}$ has been used (recall $M$ is the mass of component $\mathrm{A}$ and $M_{\odot}$ is the solar mass), and the numerical values $m_{\odot} \simeq 1.476 \times 10^{3} \mathrm{~m}, 1 \mu \mathrm{as} \simeq 4.848 \times 10^{-12} \mathrm{rad}$ and $1 \mathrm{pc} \simeq 3.086 \times 10^{16} \mathrm{~m}$ have been inserted, so that $\frac{2 m_{\odot}}{\mu \mathrm{aspc}} \simeq 0.0197$. The simplified inclination formula (D4) has first been obtained by Klioner, et al. [2003]; here the fact is noticed, that due to the divergence of the post-Newtonian solution (D1) for $d \rightarrow 0$, which corresponds to $\psi \rightarrow \pi$, the applicability of (D4) is restricted by the condition $d \gg m$. Using $d=A|\cos i|$ one obtains the validity condition for the applicability of (4):

$$
\left|\frac{\pi}{2}-i\right|_{\mathrm{KMS}} \gg \arcsin \frac{m}{A} \text {. }
$$


Equation (D4) provides a relation between inclination $i$ and light deflection $\varphi$ for a binary system characerized by it's distance $r$ from the observer and it's stellar mass $M$; note that (D4) agrees with the simplified inclination formula given by Equation (19).

\section{Appendix E: Probability Distribution}

Assume a probability distribution of a quantity $x$ is given by $f(x)$. The probability $P$, to find a system in the interval $x_{i} \leq x \leq x_{i}+\Delta x$ is given by

$$
P\left(x_{i} \leq x \leq x_{i}+\Delta x\right)=\frac{\int_{x_{i}}^{x_{i}+\Delta x} d z f(z)}{\int_{x_{\min }}^{x_{\max }} d z f(z)},
$$

where the region of validity of probability distribution $f(x)$ is given by $x_{\min }$ and $x_{\max }$. In the infinitesimal limit $\Delta x \rightarrow d x$, one obtains by series expansion the following explicit form for the here used probability distributions: for a power law $f(x) \sim x^{-\alpha}$ with $\alpha \neq 1$ one finds

$$
f(x)=\frac{(1-\alpha) x^{-\alpha}}{x_{\max }^{(1-\alpha)}-x_{\min }^{(1-\alpha)}},
$$

and for a logarithmic law $f(x) \sim x^{-1}$ one has

$$
f(x)=\frac{1}{x}\left(\ln \frac{x_{\max }}{x_{\min }}\right)^{-1} .
$$

The normalization is $\int_{x_{\min }}^{x_{\max }} f(x) d x=1$ and the averaged value $\bar{x}=\int_{x_{\min }}^{x_{\max }} f(x) x d x$. 